\title{
Caracterización de un material compuesto por suelo arenoso, ceniza de cáscara de arroz y cal potencialmente útil para su uso en pavimentación
}

\section{Characterization of a material comprised of sandy soil, rice husk ash and potentially useful lime in pavements}

\author{
Leonardo Behak*1, Washington Peres Núñez**
}

* Facultad de Ingeniería, U niversidad de la República, U RU GUAY
** U niversidade Federal do Rio Grande do Sul, BRASIL

Resumen

PAG. $34-41^{2}$

\begin{abstract}
Se presenta una investigación de caracterización de una mezcla de suelo arenoso con ceniza de cáscara de arroz (CCA) y cal. El objetivo fue estudiar las propiedades físicas y mecánicas de las mezclas, procurando encontrar una solución económica para pavimentos de bajo volumen de tránsito y resolver el problema ambiental de la disposición de la ceniza residual de la quema de la cáscara de arroz. El suelo arenoso fue extraído de las proximidades de Montevideo. Fue utilizada una CCA residual de la incineración de cáscara, realizada en un horno de una planta de parboilización de arroz localizada en la Ciudad de Treinta y Tres. Se realiza un análisis de la influencia de diferentes mezclas de suelo con CCA y cal en la composición mineralógica mediante difractogramas de rayos- $x$, parámetros óptimos de compactación, CBR, resistencia a la compresión inconfinada y a la tracción diametral y durabilidad.
\end{abstract}

Palabras Clave: Pavimentos de bajo volumen, estabilización de suelos, ceniza de cáscara de arroz

Abstract

An investigation of characterization of a sandy soil mixture with rice husk ash (RHA) and lime is presented. Their objective was to study the physic and mechanic properties of the mixtures, trying to find an economical solution for low-volume transit pavements and to solve an environmental problem like is the deposition of the residual ash from de husk rice burning. The soil was extract in the nearness of Montevideo. A residual RHA of the husk incineration, carried out in an oven of the rice parboilization plant localized in Treinta y Tres City, was used. An analysis of the influence of different mixtures of soil with RHA and lime in the mineralogical composition by $\mathrm{x}$-ray difractograms, optimum compaction parameters, CBR, unconfined compression and split tensile strength and durability is made.

Keyw ords: Low-Volume Pavements, soil stabilization, rice husk ash

\section{Introducción}

El crecimiento de la producción agrícola e industrial, trae como contrapartida el incremento de residuos, haciendo cada vez más difícil, costoso y ambientalmente insustentable su tratamiento y disposición final. Por ello es creciente en todo el mundo el interés en la utilización de los diversos residuos en distintas áreas, consiguiendo no sólo beneficios ambientales sino económicos. Particularmente en pavimentos se ha procurado utilizar productos residuales como estabilizantes

\footnotetext{
1 Autor de correspondencia / Corresponding author: Julio Herrera y Reissig 565, C.P. 11.300, M ontevideo. Uruguay. Teléfono: 711 2880, E-mail: Ibehak@fing.edu.uy
}

de suelos para la construcción de subrasantes y capas de sub-base y base.

La cáscara de arroz es el mayor residuo resultante de la producción agrícola de granos y su disposición final es uno de los mayores problemas existentes en los países productores de arroz. Cada 4 toneladas de arroz producidas, 1 tonelada es cáscara de arroz (Ali et al., 1992). Velupillai et al. (1996) estimaron que más de 100 millones de toneladas de cáscara de arroz son generadas

\footnotetext{
2 Agradecimientos / Acknowledgements:

XIII Congreso de Ingeniería de Transporte 22 al 26 de octubre de 2007

Departamento de Ingeniería de Transportes y Logística - Escuela de Ingeniería U C Sociedad Chilena de Ingeniería de Transporte (Sochitran)
} 


\section{M ateriales usados}

cada año en el mundo. En U ruguay la producción de arroz a aumentado de 391.188 ton en la zafra 1987-88 hasta 1.214.490 ton en la zafra 2004-05 (ACA, 2006), estimándose que sólo en esta última zafra se generaron 300.000 ton de cáscara. La producción anual de arroz en Brasil se ha mantenido próxima a 10.000.000 ton, siendo la cantidad de cáscara generada más significativa aun. La disposición final de tales cantidades de cáscara es un auténtico problema sin solución definitiva.

Para reducir el volumen de residuo a depositar la cáscara de arroz es quemada, intentando darle una utilidad económica, como energético en calderas de secado del propio arroz (proceso de parboilización), combustible para la producción de cemento Portland, para la generación de energía eléctrica, etc. Las diferentes alternativas de quema de cáscara de arroz traen un nuevo problema, la disposición final de la ceniza producida. Durante este proceso se volatilizan los componentes orgánicos de la cáscara: celulosa y lignina, quedando como remanente residual la ceniza. Se estima que la constitución de la ceniza de cáscara de arroz (CCA) es aproximadamente un $20 \%$ en peso de la cáscara de la cual fue quemada (Juliano, 1985). De haberse incinerado toda la cáscara de arroz en la zafra uruguaya 2004-05, se hubieran generado 60.700 ton de ceniza.

Los suelos arenosos o limosos muchas veces no presentan propiedades adecuadas como materiales de capas de base 0 sub-base de pavimentos. Sin embargo son muy frecuentes en las regiones de cultivo de arroz. Los materiales de mejor calidad se localizan lejos de los caminos vecinales de salida de la producción de arroz, resultando su uso una alternativa de elevado costo de transporte. La evaluación de la estabilización de suelos arenosos o limosos locales con adición de CCA y cal es una alternativa de interés. Siendo que la CCA está compuesta entre 90 - 96\% por sílice (Juliano, 1985), proporcionaría a los suelos la sílice necesaria para reaccionar con la cal y formar productos puzolánicos, obteniéndose así, materiales más resistentes, menos deformables y más durables.

Una investigación particular de caracterización de un suelo arenoso estabilizado con CCA y cal fue realizada con el objetivo principal de evaluar el mejoramiento de sus propiedades físicas y mecánicas, como paso previo a un estudio de verificación de desempeño de este material, así conformado, en las diferentes capas de pavimentos de bajo volumen de tránsito.
En la investigación se utilizó un suelo sedimentario arenoso, extraído de una cantera situada en las proximidades de Montevideo. Fue empleada una CCA residual de la incineración de la cáscara en un homo de una planta de parboilización del arroz, localizada en la Ciudad de Treinta y Tres, al noreste de U ruguay. Se adoptó una cal hidratada comercial, producida en U ruguay.

\subsection{Suelo}

En la Tabla 1 se presentan las características del suelo arenoso. La composición mineralógica se determinó mediante difractometría de rayos- $x$; observándose el resultado en la Figura 1. La fracción gruesa del suelo está conformada por cuarzo, mientras que la fracción fina es predominantemente caolinita y montmorillonita.

Tabla 1. Propiedades del suelo sedimentario arenoso

\begin{tabular}{|l|c|}
\hline \multicolumn{2}{|l|}{ Distribución Granulométrica } \\
\hline Grava & $1,4 \%$ \\
\hline Arena & $92,1 \%$ \\
\hline Limo & $0,9 \%$ \\
\hline Arcilla & $5,6 \%$ \\
\hline Propiedades Físicas & 0 \\
\hline Índice Plástico & 2,65 \\
\hline Gravedad Específica & \\
\hline Clasificación & SW-SM \\
\hline $\begin{array}{l}\text { Sistema Unificado de Clasificación de } \\
\text { Suelos }\end{array}$ & $\begin{array}{c}\text { A-1-b } \\
(0)\end{array}$ \\
\hline AASHTO &
\end{tabular}

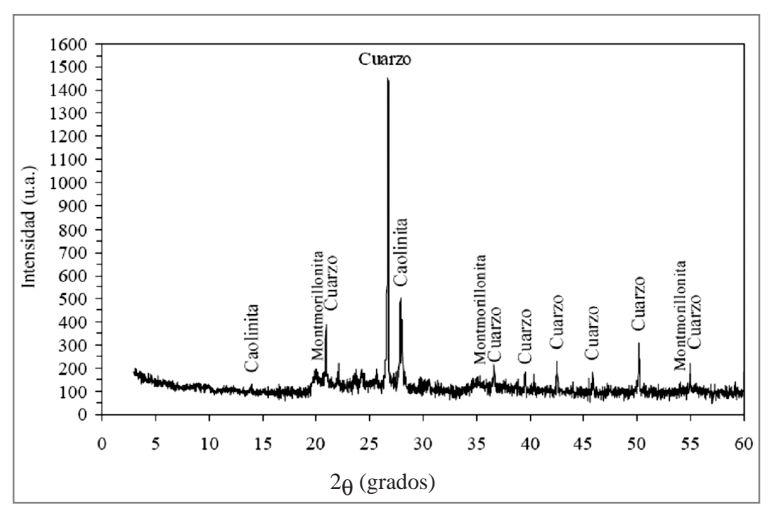

Figura 1. Difractograma de rayos-x del suelo arenoso 


\subsection{Ceniza de Cáscara de Arroz}

La ceniza de cáscara de arroz (CCA) utilizada en la investigación presenta un color negro, debido a que la temperatura de quema no es controlada. Siguiendo la propuesta de Houstin (1972), puede ser clasificada como una ceniza con alto contenido de carbón. La Tabla 2 presenta las características de la CCA.

Tabla 2. Propiedades de la Ceniza de Cáscara de Arroz

\begin{tabular}{||l|c||}
\hline \multicolumn{2}{||l}{ Distribución Granulomét } \\
\hline Pasa Tamiz No. $4(4760 \mu \mathrm{m})$ & $99,6 \%$ \\
\hline Pasa Tamiz No. $200(74 \mu \mathrm{m})$ & $11,8 \%$ \\
\hline Pasa $2 \mu \mathrm{m}$ & $0,8 \%$ \\
\hline Materia Orgánica & $18,7 \%$ \\
\hline Gravedad Específica & 1,81 \\
\hline
\end{tabular}

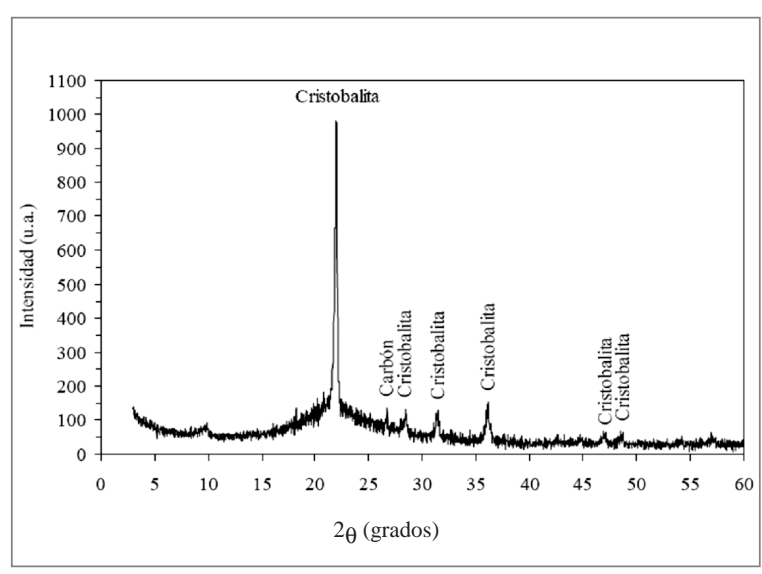

Figura 2. Difractograma de rayos-x de la ceniza de cáscara de arroz

La Figura 2 muestra la mineralogía de la CCA por medio del difractograma de rayos-x. Se observan picos característicos de cristobalita (un tipo de sílice) y de carbón, que marcan que parte de la ceniza tiene estructura cristalina, lo que afecta su actividad puzolánica. Sólo la sílice amorfa es capaz de reaccionar químicamente con los iones calcio de la cal, formando los productos cementantes que estabilizan el suelo arenoso. La presencia de carbón es otra causa más de baja actividad puzolánica de la CCA.

\section{$2.3 \mathrm{Cal}$}

La cal utilizada es de tipo cálcica, compuesta en un $66 \%$ por óxido de calcio y en un $5,3 \%$ por óxido de magnesio. Es una cal fina, pasando el $100 \%$ por el tamiz $\mathrm{N} \cong .10(2 \mathrm{~mm})$ y el $92,9 \%$ por el tamiz $\mathrm{N}$ ㅇ. 200 $(0,074 \mathrm{~mm})$.

\section{Ensayos de laboratorio}

Para el análisis del mejoramiento en las propiedades del suelo arenoso cuando se lo estabiliza con CCA y cal se realizaron ensayos de resistencia a la compresión inconfinada, de resistencia a la tracción por compresión diametral y de durabilidad. Además del suelo natural, se consideraron tres mezclas: suelo $+15 \%$ de CCA $+5 \%$ de cal, suelo $+20 \%$ de CCA $+5 \%$ de cal y suelo $+20 \%$ de CCA $+10 \%$ de cal. Las probetas fueron moldeadas, en triplicado, inmediatamente después de realizarse la mezcla del suelo con la CCA, la cal y el agua. Las probetas de suelo fueron compactadas a las condiciones óptimas del correspondiente ensayo Proctor normal, mientras que las mezclas se compactaron, adoptándose los parámetros óptimos del ensayo Proctor normal de la mezcla suelo $+20 \%$ CCA $+10 \%$ Cal. Las mezclas se dejaron curar para permitir las reacciones alcalinas, variando el tiempo de curado según el ensayo. Un análisis de las reacciones que tienen lugar en las mezclas fue realizada mediante difractogramas de rayos$x$ sobre muestras extraídas de las probetas ensayadas a resistencia a la compresión inconfinada con 28 días de cura. Fue utilizado un difractómetro de polvo con radiación CuKalfa y de longitud de onda 1,5418 $\AA$.

\subsection{Compactación}

Los ensayos de compactación Proctor normal se realizaron de acuerdo a la Norma AASHTO T99-86, utilizándose moldes de $15 \mathrm{~cm}$ de diámetro interior. Las mezclas se compactaron inmediatamente después de adicionada y homogeneizada el agua.

\subsection{Capacidad Soporte de California}

Como parámetro indicativo, se realizaron ensayos de capacidad soporte de California (CBR), sobre las mismas probetas del suelo y de la mezcla de suel $0+20 \%$ de $C C A+10 \%$ de cal con 28 días de cura, 
compactadas en el ensayo Proctor normal. Las probetas previamente al ensayo fueron sometidas a un proceso de saturación durante 96 horas y luego sometidas a carga siguiendo el procedimiento indicado en la Norma AASHTO T193-81

\subsection{Resistencia a la Compresión Inconfinada}

La resistencia a la compresión inconfinada (RCI) del suelo y de las mezclas de suelo con CCA y cal, fue determinada conforme la N orma AASHTO T20870. Los ensayos se realizaron sobre probetas curadas 7 , 14, 28 y 56 días. Las compactación se realizó en moldes metálicos tripartidos de $7,65 \mathrm{~cm}$ de altura e $3,72 \mathrm{~cm}$ de diámetro interno, por método estático. Se adoptó como valor de resistencia, la correspondiente al pico de la curva tensión-deformación.

\subsection{Resistencia a la Tracción por Compresión Diametral}

Ensayos de resistencia a la tracción por compresión diametral (RCD) se efectuaron de acuerdo con el método DNER-ME 138/94. Las probetas se compactaron en moldes de $10,14 \mathrm{~cm}$ de diámetro interno y $6,39 \mathrm{~cm}$ de altura, inmediatamente después de hecha la mezcla. Se hizo un análisis para 14, 28 y 56 días de cura.

\subsection{Durabilidad}

Para evaluar el efecto del medio ambiente sobre las mezclas de suelo con CCA y cal, se realizaron ensayos de durabilidad. Se utilizó el método propuesto por Hoover et al. (1958) que evalúa la durabilidad a ciclos de humedecimiento y secado, midiéndose la pérdida de resistencia a la compresión inconfinada. Se compactaron estáticamente probetas de $7,65 \mathrm{~cm}$ de altura y $3,72 \mathrm{~cm}$ de diámetro que luego de 28 días de cura, se sometieron a 12 ciclos de humedecimiento y secado. Cada ciclo comprendió un período de 48 horas, siendo $24 \mathrm{~h}$ de inmersión en agua y $24 \mathrm{~h}$ de secado al aire. 0 tras probetas de cada mezcla permanecieron almacenadas en cámara húmeda durante los 12 ciclos. Finalmente, las probetas sometidas y no sometidas a ciclos fueron ensayadas a la RCI. Un material se durable frente a la acción del humedecimiento y secado cuando la resistencia de las probetas sometidas a ciclos respecto a la resistencia de las probetas no sometidas a ciclos es al menos del 80\% (Marcon, 1977).

\section{Análisis de resultados}

La Figura 3 muestra los difractogramas de rayos$x$ de las tres mezclas de suelo con CCA y cal con 28 días de cura. Los picos correspondientes al cuarzo y a la cristobalita se mantienen en las tres mezclas, debido a que el cuarzo de la fracción gruesa del suelo arenoso y la cristobalita cristalina de la CCA no pueden reaccionar con la cal.

En las mezclas de suelo $+20 \%$ CCA $+5 \%$ Cal y suelo $+20 \%$ CCA $+10 \%$ Cal se observan nuevos picos. La combinación de $15 \%$ de CCA y $5 \%$ de cal sería insuficiente para formar productos cementantes en cantidades adecuadas como para ser identificados en el difractograma. Nuevos picos de antigorita, un silicato hidratado de magnesio, se formaron en la mezcla de suelo $+20 \% \mathrm{CCA}+5 \% \mathrm{Cal}$, mientras que nuevos picos de portlandita, un hidróxido de calcio, son identificados en la mezcla de suelo $+20 \%$ CCA $+10 \%$ Cal. Ambos minerales son asociables a productos cementantes, indicando que al cabo de 28 días de cura, han tenido lugar reacciones entre la sílice amorfa de la CCA y los iones calcio de la cal hidratada. Los picos de caolinita y montmorillonita desaparecen en las mezclas, probablemente debido a reacciones entre éstos y parte de la cal, colaborando a la formación de productos cementantes.

\subsection{Compactación}

Las curvas de compactación del suelo y de la mezcla de suelo $+20 \% \mathrm{CCA}+10 \% \mathrm{Cal}$ del ensayo Proctor normal se presentan en la Figura 4. Ambas curvas son achatadas, sin pico definido, típico de los suelos arenosos, ya que el peso específico seco de este tipo de suelos no varía mucho entre su estado más suelto y más denso y porque el método de aplicación de energía por impacto del ensayo Proctor no es el más adecuado para compactarlos. En la mezcla de suelo con CCA y cal, al realizarse la compactación inmediatamente después de la adición de agua y de la homogeneización, no hay modificaciones considerables en su textura. Las reacciones entre la ceniza y la cal son lentas, dependientes del tiempo, mientras que el bajo contenido de fracción fina del suelo hace que las posibles reacciones rápidas entre los minerales arcillosos y la cal sean prácticamente despreciables. 


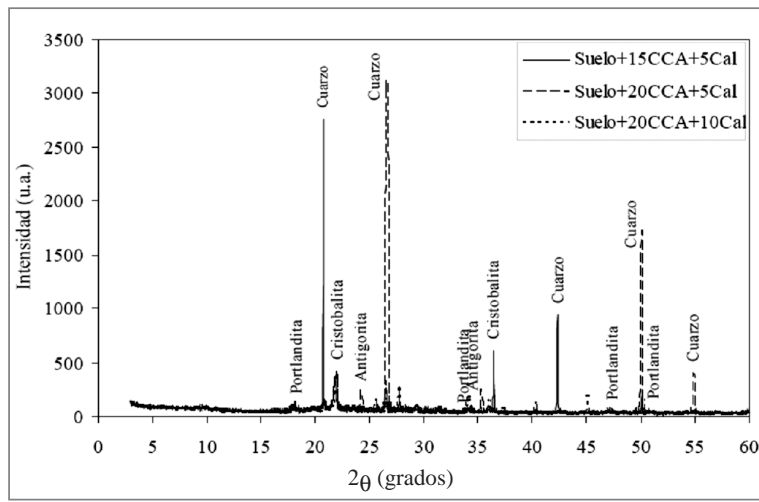

Figura 3. Difractogramas de rayos-x de las mezclas de suelo con CCA y cal con 28 días de cura

Respecto al suelo sin tratar, la adición de $20 \%$ de CCA y $10 \%$ de cal causa una fuerte disminución del peso específico seco máximo y un aumento de la humedad óptima. La disminución del peso específico seco máximo de la mezcla se debe a la suma de las bajas gravedades específicas de la CCA y de la cal, sendo que no hubo tiempo suficiente para el desarrollo de reacciones puzolánicas. Parte del agua adicionada a la mezcla es absorbida por la CCA, debido a sus características porosas (Zhang et al., 1996). O tra parte del agua es consumida por la cal en la hidratación. Como resultado, para conseguir la mayor eficiencia en la compactación, será necesario adicionar más agua para reducir los efectos de la succión.

\subsection{Capacidad Soporte California}

El CBR no es apropiado para caracterizar la resistencia de suelos estabilizados (Thompson, 1975). Los valores de CBR para mezclas curadas pueden ser superiores al $100 \%$. En esos casos es notorio que el resultado del ensayo sólo sirve como indicativo general de resistencia. La Figura 5 muestra los comportamientos del CBR del suelo y de la mezcla de suelo $+20 \%$ CCA $+10 \%$ Cal, curada 28 días en función de la humedad de compactación de las probetas ensayadas.

Los valores de CBR de la mezcla son mayores que los del suelo para todas las humedades de compactación. También se observa un aumento del CBR de la mezcla, inclusive para las humedades de compactación superiores a la óptima (8,5\%). Lo mismo sucede con los CBR del suelo aunque la razón de aumento disminuye gradual mente a medida que aumenta la humedad de compactación. Para las humedades óptimas de compactación, el valor de CBR del suelo fue $9 \%$ y el de la mezcla suelo $+20 \%$ CCA $+10 \%$ Cal fue de
$25 \%$, es decir, se observa un aumento de 2,8 veces en el CBR de la mezcla con relación al del suelo.

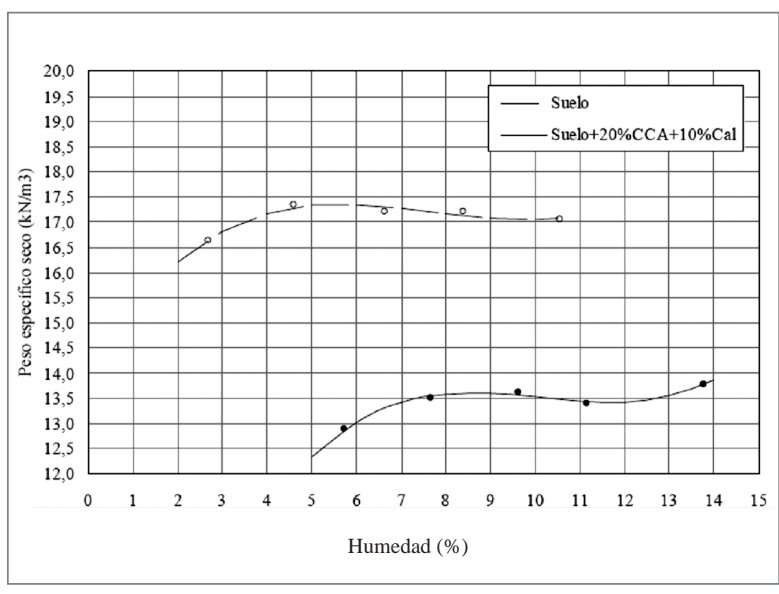

Figura 4. Curvas de compactación del suelo y del suelo $+20 \%$ de $\mathrm{CCA}+10 \%$ de cal

El significativo incremento de CBR en la mezcla, indicaría que existen reacciones puzolánicas entre la sílice amorfa de la CCA y los iones calcio de la cal, produciendo mejoras de la capacidad soporte del suelo estabilizado. El efecto de filler de la ceniza y de la cal adicionados al suelo, actuando como finos, provocan una corrección granulométrica, siendo otra causa posible de aumento de CBR. En función del valor de CBR obtenido para la mezcla, se puede afirmar que ese material podría ser empleado hasta como capa de subbase de pavimentos.

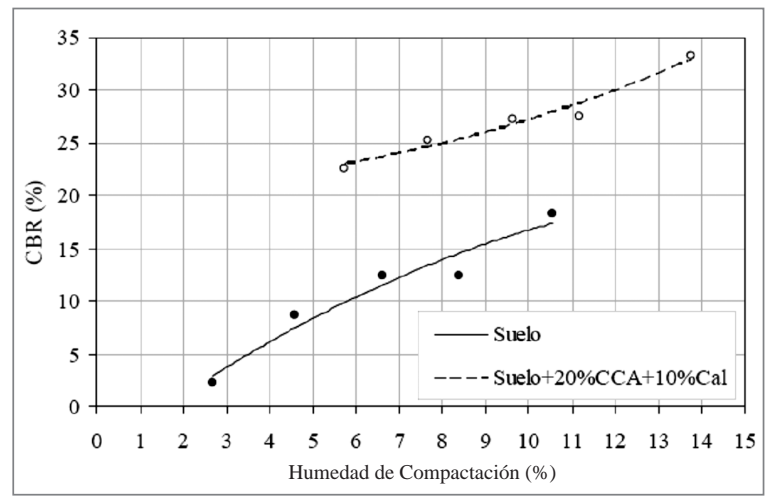

Figura 5. CBR vs. humedad de compactación del suelo y del suelo $+20 \%$ CCA $+10 \%$ Cal

\subsection{Resistencia a la Compresión Inconfinada La Figura 6 muestra la evolución de la $\mathrm{RCI}$ con}


el tiempo de cura de las mezclas de suelo con CCA y cal. En el origen está colocada la $\mathrm{RCl}$ del suelo sin tratar, cuyo valor es de $14 \mathrm{kPa}$. Se observa un aumento constante de la RCI para las tres mezclas, con una mayor razón para los tiempos de cura más bajos. El mayor valor de $\mathrm{RCl}$ corresponde a la mezcla suelo $+20 \% \mathrm{CCA}+10 \% \mathrm{Cal}$ con 56 días de cura, siendo de $276 \mathrm{kPa}, 20$ veces mayor que la $\mathrm{RCl}$ del suelo natural. Con 56 días de cura, las curvas correspondientes a $5 \%$ de cal (con 15 o 20\% de CCA) muestran una tendencia a estabilizarse, sugiriendo que toda la cal reaccionó con la sílice disponible en la ceniza. La pendiente de la curva de la mezcla de suelo $+20 \%$ CCA $+10 \%$ Cal indicaría un probable aumento de la $\mathrm{RCl}$, posterior a los 56 días, debido a que habría suficiente cantidad de cal como para reaccionar con la sílice amorfa de la ceniza.

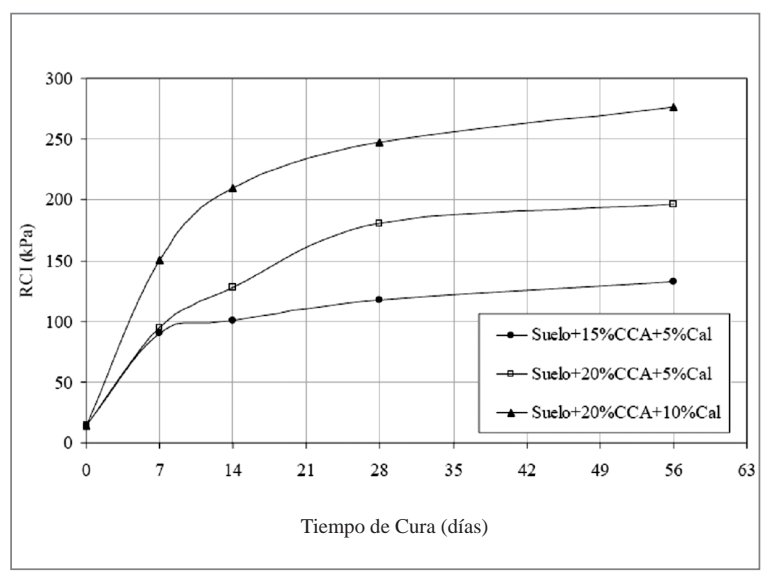

Figura 6. RCI vs. tiempo de cura para diferentes mezclas de suelo con CCA y cal

La $\mathrm{RCl}$ aumenta con el aumento de los contenidos de CCA y cal, siendo mayor la razón de aumento cuando se pasa de $5 \%$ a $10 \%$ de cal, manteniendo un $20 \%$ de CCA, que cuando se pasa de $15 \%$ a $20 \%$ de CCA, manteniendo un $5 \%$ de cal. El aumento de la $\mathrm{RCl}$ a creciente contenido de CCA y a contenido de cal constante, se debería a que el aumento de CCA proporcionaría mayor cantidad de sílice amorfa capaz de reaccionar con la cal, favoreciendo las reacciones cementantes, aunque estas sean muy lentas. El incremento de cal, no sólo aumenta considerablemente la $\mathrm{RCl}$ sino también acelera las reacciones con la sílice amorfa de la ceniza, alcanzando mayores $\mathrm{RCl}$ en las edades prematuras.
4.4 Resistencia a la Tracción por Compresión Diametral La evolución de la RCD con el tiempo de cura del suelo estabilizado con diferentes contenidos de CCA y cal es mostrada en la Figura 7. La RCD aumenta notablemente, disminuyendo la tasa de incremento con el aumento del tiempo de cura. La máxima RCD corresponde a la mezcla suelo $+20 \%$ CCA $+10 \%$ Cal con 56 días de cura, con un valor de $95 \mathrm{kPa}$. Coincidentemente con las conclusiones de N úñez (1991), el tiempo de cura es extremadamente importante para el desarrollo de RCD en las mezclas de suelo con CCA y cal. Siendo que los suel os naturales no tienen resistencia a la tracción, el surgimiento de RCD cuando se estabiliza el suelo con CCA y cal indica la generación de productos cementantes debido a las reacciones de los iones calcio de la cal con la sílice amorfa de la ceniza.

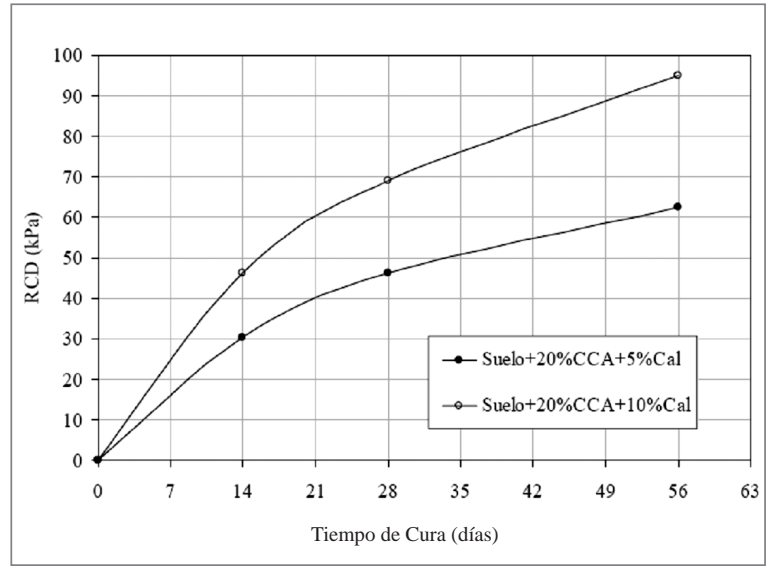

Figura 7. RCD vs. tiempo de cura para diferentes mezclas de suelo con CCA y cal

\subsection{Durabilidad}

La influencia de los ciclos de humedecimiento y secado, evaluada como el cociente entre la $\mathrm{RCl}$ entre las muestras sometidas a ciclos y las muestras no sometidas a ciclos ( $\mathrm{Cr}$ ), es indicada en la Tabla 3. Se observa un aumento de $\mathrm{Cr}$ con el aumento de los contenidos de CCA y de cal. Además, Ia RCI de la mezcla con $20 \%$ de CCA y $10 \%$ de cal sometida a ciclos es mayor que las $\mathrm{RCI}$ de las mezclas con $15 \%$ de CCA e $5 \%$ de cal y con $20 \%$ de CCA y $5 \%$ de cal no sometidas a ciclos.

La durabilidad, aun siendo inferior al $80 \%$ propuesto por Marcon (1977), puede ser considerada aceptable para la mezclas suelo $+20 \%$ CCA $+5 \%$ Cal y 
suelo $+20 \%$ CCA $+10 \%$ Cal, si se considera la baja actividad puzolánica de la CCA utilizada y pensando en la utilización de estos materiales en pavimentos de bajo a mediano volumen de tránsito.

Tabla 3. Cociente de resistencia $(\mathrm{Cr})$ para diferentes mezclas de suelo con CCA y cal

\begin{tabular}{|c|c|c|c|}
\hline \multirow{2}{*}{$\begin{array}{c}\text { Contenidos } \\
(\% \mathrm{CCA}+\% \mathrm{Cal})\end{array}$} & Sin Ciclos & $\mathrm{Cr}$ \\
\cline { 2 - 4 } & RCI (kPA) \\
\hline $15+5$ & 210 & Con Ciclos & 64 \\
\hline $20+5$ & 197 & 134 & 72 \\
\hline $20+10$ & 364 & 141 & 77 \\
\hline
\end{tabular}

\section{Conclusiones}

- Los difractogramas de rayos x confirman que, al cabo de 28 días de cura, se forman productos cementantes cuando reaccionan los iones de calcio de la cal con la sílice amorfa de la CCA. Estos productos son los responsables de la estabilización del suelo arenoso.

- Se observó un importante aumento del CBR de la mezcla de suelo con $20 \%$ de CCA y $10 \%$ de cal, con 28 días de cura, respecto al del suelo natural. El valor de CBR obtenido para la mezcla fue de $25 \%$, lo que permite afirmar que el material así producido podría ser empleado como capa de subbase de pavimentos.

- La RCI de las mezclas aumenta al aumentar el contenido de CCA y de cal y el tiempo de cura, siendo el valor máximo de $276 \mathrm{kPa}$, correspondiente a la mezcla suelo $+20 \%$ CCA $+10 \%$ Cal con 56 días de cura, 20 veces mayor que para el suelo natural. La razón de aumento es mayor para los tiempos de cura más bajos, siendo sus valores mayores cuanto más CCA y cal son adicionados. Un mayor contenido de CCA proporcionaría suficiente sílice amorfa para las reacciones con la cal, mientras que el aumento del contenido de cal, además acelera estas reacciones proporcionando mayor $\mathrm{RCl}$ en las edades prematuras.

- La RCD de las mezclas aumenta al aumentar el contenido de CCA y de cal y el tiempo de cura. El máximo valor de RCD correspondió a la mezcla suelo $+20 \%$ CCA $+10 \%$ Cal con 56 días de cura, siendo de $95 \mathrm{kPa}$. La existencia de RCD en las mezclas de suelo con CCA y cal indica la generación de productos cementantes debido a las reacciones entre la cal y la ceniza.

- La durabilidad determinada en las mezclas de suelo con CCA y cal, puede considerarse aceptable si se tiene en cuenta la baja actividad puzolánica de la CCA investigada y se piensa en la utilización de estos materiales en pavimentos de bajo a mediano volumen de tránsito.

Los materiales compuestos de suelos arenosos, CCA y cal, son potencialmente utilizables para la construcción de capas de pavimentos de bajo a mediano volumen de transito, con posibles beneficios económicos y ambientales, al habilitar el uso de suelos arenosos locales con reducción de costos de transporte y el aprovechamiento de un residuo como es la ceniza de cáscara de arroz.

\section{Agradecimientos}

Los autores agradecen al Prof. Álvaro Rostan, En nombre del Departamento de Geotécnica de la Universidad de la República del U ruguay por el apoyo logístico y financiero de los trabajos de campo y de laboratorio. A la CAPES de Brasil y al Programa de PósGraduação em Engenharia Civil de la UFRGS, que posibilitaron la realización de la maestría de Leonardo Behak, base de este trabajo. Particularmente al Prof. Jorge A. Ceratti.

\section{Referencias}

ACA (2006), Evolución del cultivo de Arroz en U ruguay. www.aca.com.uy/datos_estadisticos/evolucion_culti vo.htm. Sitio visitado en junio de 2007.

Ali F. H., Adnan A. y Choy C.K. (1992), Geotechnical Properties of a Chemically Stabilized Soil from Malaysia with Rice Husk Ash as an Additive. Geotechnical and Geological Engineering, Vol 10, 117 - 134. Hoover J. M, Handy R.L., Davidson D.T. (1958), Durability of Soil-Lime-Fly Ash Mixtures Compacted above Standard Proctor Density. Highway Research Board Bulletin, No 139, 1-11.

Houston D. F. (1972), Rice Hulls. Rice: Chemistry and 
Technology, American Association of Cereal Chemists, 301-340.

Juliano B. O . (1985), Rice: Chemistry and Technology. American Association of Cereal Chemists. Marcon A. F. (1977), Durabilidade e Módulo de Elasticidade de Misturas Areia-Cal-Cinza Volante. Disertação de Mestrado, U niversidade Federal de Rio de Janeiro.

Núñez W. P. (1991), Estabilização Físico-Química de um Solo Residual de Arenito Botucatú, Visando seu Emprego na Pavimentação. Disertação de M estrado, Curso de Pós-Graduação em Engenharia Civil, Universidade Federal de Rio Grande do Sul, 150 p. Thompson M. R. (1975), Soil-Lime Mixtures for Construction of Low-Volume Roads. Transportation Research Board Special Report, No 160, 149-165. Velupillai L., Mahin D.B., Warshaw J.W., y Wailes E.J. (1996), A Study of the Market for the Rice-Husk-toEnergy Systems and Equipment. Louisiana Agricultural Centre, p. 24.

Zhang M. H., Lastra R., y Malhotra V.M. (1996), Rice Husk Ash as Paste and Concrete: Some Aspects of $\mathrm{Hydration}$ and the Microstructure of the Interfacial Zone between the Aggregate and Paste. Cement and Concrete Research, Vol 26, 963-977. 